\title{
A Review of Superficial Femoral Artery Angioplasty and Stenting
}

Rami O Tadros*, Ageliki G Vouyouka, Windsor Ting, Victoria Teodorescu, Sung Yup Kim, Michael L Marin and Peter L Faries

Department of Surgery, The Icahn School of Medicine at Mount Sinai, New York, USA

*Corresponding author: Rami O Tadros, Department of Surgery, The Icahn School of Medicine at Mount Sinai, New York, USA, Tel: 3478520741; Email: rami.tadros@mountsinai.org

Rec date: Jan 27, 2015; Acc date: Feb 06, 2015; Pub date: Feb 08, 2015

Copyright: $\odot 2015$ Tadros RO, et al. This is an open-access article distributed under the terms of the Creative Commons Attribution License, which permits unrestricted use, distribution, and reproduction in any medium, provided the original author and source are credited.

\section{Abstract}

Introduction: Peripheral arterial disease (PAD) of the superficial femoral artery (SFA) is the most common cause of intermittent claudication. Several endovascular treatment options exist; the most frequent are angioplasty or angioplasty with stent placement. The aim of this review is to clarify the role of angioplasty alone vs. angioplasty with stent placement.

Methods: A literature review was conducted reviewing the topic of SFA angioplasty and SFA angioplasty with stenting. Three notable randomized trials were included in addition to other available studies on the topic.

Results: When a residual stenosis or flow limiting dissection is visualized, stenting is clearly necessary. Current literature favors the use angioplasty for short $(\sim 4 \mathrm{~cm})$ SFA stenoses or occlusions due to comparable patency and results. Primary stenting using Nitinol stents may be a superior initial treatment for intermediate $(6-8 \mathrm{~cm})$ and long $(>10 \mathrm{~cm})$ length lesions. In these intermediate and long length lesions, the use of stents appears to reduce the restenosis rates and improves patency.

Conclusion: Angioplasty works best for short SFA stenoses or occlusions. However, angioplasty with stenting using is likely superior initial treatment for intermediate and long length lesions.

Keywords: Angioplasty; Peripheral arterial disease; Artery; Endovascular

\section{Introduction}

Peripheral arterial disease (PAD) of the superficial femoral artery (SFA) is the most common cause of intermittent claudication. Atherosclerotic disease of the SFA is localized to the region of Hunter's canal. An isolated occlusion or stenosis of the SFA often results in decreased perfusion of the leg, resulting in demand related, reversible, ischemic pain localized to the calf. Ischemic rest pain and tissue loss, also known as critical limb ischemia (CLI), are uncommon manifestations of isolated SFA disease. CLI is more commonly observed when occlusive disease of the SFA is combined with occlusive disease involving the below knee popliteal artery or tibial arteries.

Endovascular treatment of the SFA was first described by Charles Dotter in 1964. In Dotters original description, he used Teflon coated dilators to sequentially angioplasty the SFA in an 82-year-old woman to treat critical limb ischemia that was considered non-operable [1]. Subsequently, Gruntzig popularized the concept of catheter directed balloon angioplasty [2]. Angioplasty disrupts the atherosclerotic plaque by displacing it radially. This action results in stretching of the adventitia thereby increasing the lumen diameter in the treated vessel. By definition, a dissection is created and if significant, can be flow limiting. Currently, the most commonly utilized endovascular revascularization options are percutaneous transluminal angioplasty (PTA) with provisional stenting or primary stenting. Provisional or selective stenting is indicated for the treatment of flow limiting dissections and/or persistent, hemodynamically significant stenoses or recoil after PTA. This approach is recommended by the Tran-Atlantic
inter-Society Consensus document II (TASC II) when treating SFA disease [3]. However, both PTA alone and primary stenting can successfully treat SFA disease. Therefore, the debate continues as to which endovascular treatment is superior.

PTA has the advantage of being inexpensive and technically simpler than primary stenting. PTA alone avoids using foreign bodies that may be a potential stimulus for intimal hyperplasia. Another benefit of using PTA alone includes avoiding material fatigue and fractures associated with stenting. Stent fatigue and fracture may result from the torque and deformation of the femoropopliteal arteries that occurs during flexion of the knee joint. Further, the advent of lower profile angioplasty balloons allows PTA of the SFA through vascular sheaths as small as 4 French. Smaller sheath diameters result in fewer complications, and therefore, are considered safer. In addition, the ease of re-intervention or bypass of an angioplastied arterial segment following PTA may be advantageous. The presence of a stent may impede endovascular re-intervention if a re-stenosis results in occlusion of the stented arterial segment. Moreover, angioplasty preserves collateral vessels that may be compromised by stent placement.

\section{Indications}

Absolute indications to treat chronic limb ischemia arise when it results in the limb threatening conditions of rest pain and tissue loss (Rutherford category 4, 5 or 6, Figure 1) [4]. Debilitating symptoms of intermittent claudication (Rutherford category 2 or 3, Figure 1) are relative, but common indications for treatment. Intermittent claudication is the most common manifestation of isolated SFA arterial disease. Though SFA disease is often present in patients with 
CLI, it is frequently seen in conjunction with multi-level arterial occlusive disease.

Once revascularization is indicated, the decision to proceed with an endovascular approach compared to an open surgical approach is frequently based on the TASCII recommendations (Figure 2) [3]. According to the recommendations, TASC-A and B lesions are managed with endovascular techniques in most cases. TASC-C lesions are treated by either bypass or endovascular revascularization based on an individual risk-benefit analysis. Generally, TASC-D lesions are surgically managed. For patients with non-operable severe claudication due to the anatomic distribution of disease or medical comorbidities, only medical management is appropriate. Amputation may be the only therapeutic option for patients with CLI whom are not candidates for revascularization. Amputation may palliate ischemic rest pain and is necessary in the setting of significant tissue loss and gangrene. Endovascular revascularization allows treatment of arterial disease in patients that would otherwise not be considered candidates for surgical bypass based on medical risk factors. This advancement has been associated with the national trend towards decreasing amputation rates [5].

\begin{tabular}{|c|c|}
\hline Classification of Chronic Limb Ischemia \\
\hline Rutherford Category & Characteristic \\
\hline 0 & Asymptomatic \\
\hline 1 & Mild claudication \\
\hline 2 & Moderate claudication \\
\hline 3 & Severe claudication \\
\hline 4 & Pain at rest \\
\hline 5 & Minor tissue loss (ischemic ulcer) \\
\hline 6 & Major tissue loss (gangrene) \\
\hline
\end{tabular}

Figure 1: Rutherford classification of chronic limb ischemia

\section{Technique}

PTA of the SFA, like all percutaneous vascular interventions, begins with vascular access. The most common access vessel is the common femoral artery (CFA). In select cases, the brachial artery can also be used. The safest approach to obtaining vascular access is by utilizing ultrasound guidance and an echogenic micropuncture needle. This technique limits the number of needle passes. Furthermore, this approach is particularly useful in patients with concomitant inflow disease whom may have diminished femoral pulses. When accessing the CFA, the puncture site must be below the inguinal ligament and above the femoral bifurcation in the region overlying the femoral head. The femoral head provides a solid surface for firm compression of the CFA necessary for hemostasis following arterial puncture. This puncture site is confirmed fluoroscopically. Fluoroscopically, the image intensifier centers over the femoral head to avoid a parallax effect. Most frequently, the puncture site is directly over the inferior third of the femoral head. After arterial puncture, the point of access is confirmed angiographically. Angiographically, the ideal entry position is between the origin of the inferior epigastric artery or lateral circumflex artery and femoral bifurcation. Access outside of this target zone is associated with a greater access site complication rate [6].

\section{Tran-Atlantic inter-Society Consensus II Classification of Femoropopliteal Disease}

\begin{tabular}{|l|ll|}
\hline $\begin{array}{l}\text { Lesion } \\
\text { Type }\end{array}$ & Stenosis or occlusion pattern & Procedure \\
\hline A & - Single stenosis $\leq 10 \mathrm{~cm}$ or occlusion $\leq \mathbf{5 c m}$. & Endovascular \\
\hline B & $\begin{array}{l}\text { - Multiple stenoses or occlusions } \leq 5 \mathrm{~cm} \text { or a single } \\
\text { severely calcified occlusion } \leq 5 \mathrm{~cm} . \\
\text { - Single stenosis or occlusion } \leq 15 \mathrm{~cm} \text { not involving the } \\
\text { below knee popliteal artery. }\end{array}$ & Endovascular \\
\hline & $\begin{array}{l}\text { - Single or multiple lesions in conjunction with occluded } \\
\text { proximal infra-geniculate vessels to improve inflow for } \\
\text { distal bypass. }\end{array}$ & \\
\hline & - Single popliteal artery stenosis. & \\
\hline - Multiple stenoses or occlusions adding to $>15 \mathrm{~cm}$ & $\begin{array}{l}\text { Endovascular } \\
\text { irrespective of calcification. }\end{array}$ \\
\hline - Two failed attempts at endovascular revascularization. \\
C
\end{tabular}

Figure 2: Tran-Atlantic inter-Society Consensus II (TASC II) Classification of Femoropopliteal Disease. CFA, Common Femoral Artery. SFA, Superficial Femoral Artery

Arterial access can be obtained ipsilateral or contralateral to the target lesion. Commonly, contralateral vascular access is preferred. Contralateral access requires an up-and-over technique to cross the aortic bifurcation. This approach is preferred because it allows an adequate working length to image and treat sequential lesions within the entire target extremity. Once contralateral access is obtained, a guide wire is advanced retrograde through the iliac arteries and abdominal aorta under fluoroscopic guidance. If using a micropuncture sheath for initial access, the 0.018 -inch guide wire is exchanged for a 0.035 -inch guide wire. The micropuncture sheath is then exchanged for an appropriately sized working vascular sheath. For SFA angioplasty, sheath sizes can range from 4 to 7 French. The smallest working sheath needed to complete the procedure should be selected. Vascular sheaths protect the access vessel during catheter manipulation and wire exchanges. Once in place, the vascular sheath should be flushed with heparinized saline.

Through the vascular sheath, a flush catheter is advanced over a guide wire into the abdominal aorta and positioned at approximately the L1 vertebra. Digital subtraction angiography (DSA) of the aorta and iliac arteries is performed unless this area has been previously evaluated by a computed tomographic angiography (CTA), magnetic resonance angiography (MRA), or a prior DSA. Pre-procedural CTA and MRA are increasingly being performed for diagnosis and preprocedure planning. CTA and MRA are of particular benefit when the femoral pulses are absent. Without pre-procedural imaging, diagnostic angiography must be used to assess the inflow. Moreover, it helps plan and facilitate crossing the aortic bifurcation into the contralateral extremity. Using a reverse curved catheter and a 0.035 -inch guide wire, the aortic bifurcation is traversed. Once the ostium of the contralateral iliac artery is engaged, the catheter and guide wire are pulled down as a single unit. This movement will secure the catheter at the aortic bifurcation. The guide wire is then advanced distally to the level of the CFA providing adequate purchase to advance a selective catheter to 
the distal external iliac artery. Selective angiograms are performed on the target extremity. The angiogram assesses the target lesion and the runoff. Patency of the runoff vessels should be assessed prior to any intervention. In addition to the runoff angiogram, the pre-procedural physical exam must document the presence or absence of pulses and/or Doppler signals. This assessment is very important because distal vessel occlusions secondary to embolization following SFA angioplasty can occur. In the presence of a single runoff vessel, an embolic protection filter can be used prior to angioplasty.

Once the decision to treat a contralateral arterial stenosis or occlusion is made, the short vascular sheath must be exchanged for a long vascular sheath using the stiff guide wire. The long vascular sheath is positioned sufficiently proximal to the treatment area. The long sheath provides stable working access in the contralateral extremity that will allow continued angiographic imaging during revascularization.

Prior to any intervention, patients are anticoagulated typically using a bolus of $80-100$ units $/ \mathrm{kg}$ of intravenous heparin. Alternative anticoagulant agents that directly inhibit thrombin, e.g., hirudin, bivalirudin, lepirudin, and argatroban, are also available. The activating clotting time (ACT) is checked every 30-60 minutes. The anticoagulant is re-dosed as needed to maintain an ACT above 250 seconds. When heparin is contraindicated, direct thrombin inhibitors are used. An advantage of heparin compared to direct thrombin inhibitors is that if access site bleeding becomes a problem, heparin can be reversed with protamine sulfate 1 to $1.5 \mathrm{mg}$ intravenously per 100 units of heparin used.

Once anticoagulated, the target SFA lesion must be traversed. Crossing the lesion is accomplished by combining a directional catheter and a 0.035 -inch guide wire. Commonly, a hydrophilic guide wire is utilized. The catheter is positioned just proximal to the target lesion providing wire support and pushability to cross the stenosis or occlusion. Crossing the lesion via the true lumen is often preferred in the majority of patients. If a 0.035 -inch hydrophilic guide wire cannot pass the lesion, then varying 0.018 -inch or 0.014 -inch guide wire and catheter combinations can be attempted. If still unable to traverse the lesion, then crossing the lesion subintimally may be possible. This technique is most effective when the vessel beyond the stenosis or occlusion is relatively normal. A normal distal vessel will facilitate reentry into the true lumen beyond the lesion. When re-entry in to the true lumen cannot be accomplished using a hydrophilic guide wire, a re-entry device may be employed. Subintimal angioplasty can be used effectively with excellent technical success and acceptable patency [7-9]. Once a wire has passed the lesion, it is advanced distally. This action will provide additional wire purchase for advancing a balloon catheter.

After crossing the lesion, an appropriate angioplasty balloon must be selected. For the purpose of arterial angioplasty, non-compliant balloons are recommended because they inflate to a uniform diameter regardless of the amount of pressure introduced into the balloon. Therefore, non-compliant balloons are less likely to cause injury to the native vessel and are more effective in treating atherosclerotic lesions. Balloon catheters can be over-the-wire or monorail. Over-the-wire balloon catheters have more pushability and are often better for crossing tight lesions. Moreover, these balloons can also be used as a catheter. Monorail balloons are less cumbersome and easier to handle because they utilize a shorter wire. Moreover, the monorail design will allow more expedient balloon catheter exchanges. However, these advantages come at the expense of pushability. Therefore, crossing a tight stenosis or occlusion using a monorail balloon can be more challenging. The diameter of a normal SFA segment beyond the lesion is measured to select the appropriate size angioplasty balloon. After selecting the appropriate balloon diameter, the ideal balloon length is chosen. The lesion length is measured using an external radiopaque ruler or a calibrated catheter. The proper length balloon must treat the target lesion without disruption of the normal vessel proximal and distal to the lesion.

Once selected, the appropriate angioplasty balloon is then advanced over the guide wire into the treatment area. Angioplasty is performed by inflating the balloon. The nominal pressure of the balloon is the pressure at which the balloon will achieve the manufactures stated diameter. With non-compliant angioplasty balloons, a greater pressure will not result in an increased balloon diameter. However, with semicompliant angioplasty balloons, greater pressures will over inflate the balloon to a larger diameter. Balloon over-sizing and/or over-inflation can cause arterial injury or significant dissections. The rated burst pressure (RBP) is the pressure at which $99.9 \%$ of balloons will not rupture. This pressure should not be exceeded. Balloon rupture can occur due to over inflation or coarse atherosclerotic plaque particularly when calcified. This complication may cause embolization of balloon fragments or air when balloons are not prepped properly. This catastrophe can result in distal ischemia. Potential air embolization can be minimized by properly preparing the balloon. Balloons are prepared by drawing the air out of the balloon in exchange for 0.50 strength contrast using a $20 \mathrm{ml}$ syringe.

When performing an angioplasty, the balloon catheter is centered within the narrowed segment of the target stenosis or occlusion. This position will focus the inflated balloon's vector forces on the tightest arterial segment. Using an insufflator, the balloon is inflated to the nominal pressure or until the balloon reaches its profile (Figure 3-A and 3-B). Normally, balloons are inflated to the nominal or rated burst pressure. As the balloon is inflated, a narrowing or "waist" will be seen at the site of stenosis or occlusion. A resolution of the "waist" may be visualized as the inflated balloon overcomes the stenosis or occlusion. When planning the use of angioplasty without stent placement, longer balloon inflation times are often used to stabilize the luminal surface of the arterial segment being treated. The longer inflation times may reduce the likelihood of a flow limiting dissection. Balloon inflations may be maintained for 30-180 seconds. After angioplasty, a repeat angiogram is performed (Figure 3-C). The presence of a persistent stenosis, vessel recoil, or a flow limiting dissection is followed by repeat angioplasty. Increasing the balloon inflation time under such circumstances is suggested. For persistent stenoses that do not resolve with standard non-compliant balloons, cutting balloons can be used. Cutting balloons are reinforced with microtomes that provide a leading edge to cut through stiff fibrotic lesions at lower pressures. This treatment is potentially safer than using a larger caliber PTA balloon and may be associated with less hemodynamically significant dissections. When a flow limiting dissection or a greater than $50 \%$ residual stenosis persists after PTA, provisional or "bailout" stenting is performed. In an attempt to limit intimal hyperplasia, cryoplasty (cold induced apoptosis) and drug eluting (paclitaxel or sirulimus releasing) balloons have been used, but their efficacy is still being assessed.

\section{Follow-up}

Postoperative follow-up for patients undergoing SFA angioplasty is routinely performed with ankle-brachial index (ABI) determination and arterial duplex ultrasound first within one month of the 
intervention, then at three to six month intervals for the first year, and annually thereafter. Loss of patency is defined as an occlusion or the presence of a hemodynamically significant stenosis at the previously treated target lesion. Occlusion is determined by a pre-occlusive blunted Doppler waveform or "pre-occlusive thump" and no flow on arterial duplex ultrasound. CTA and DSA can also be used to diagnose or confirm an occlusion. A hemodynamically significant stenosis is defined by a reduction in the arterial lumen diameter by greater than $50 \%$. Significant stenoses are determined by a decreased ABI of greater than 0.15 , duplex ultrasound demonstrating a PSV more than double the proximal segment or PSV greater than $200 \mathrm{~cm} / \mathrm{sec}$ with a monophasic waveform. CTA or DSA can also help determine the degree of stenosis.

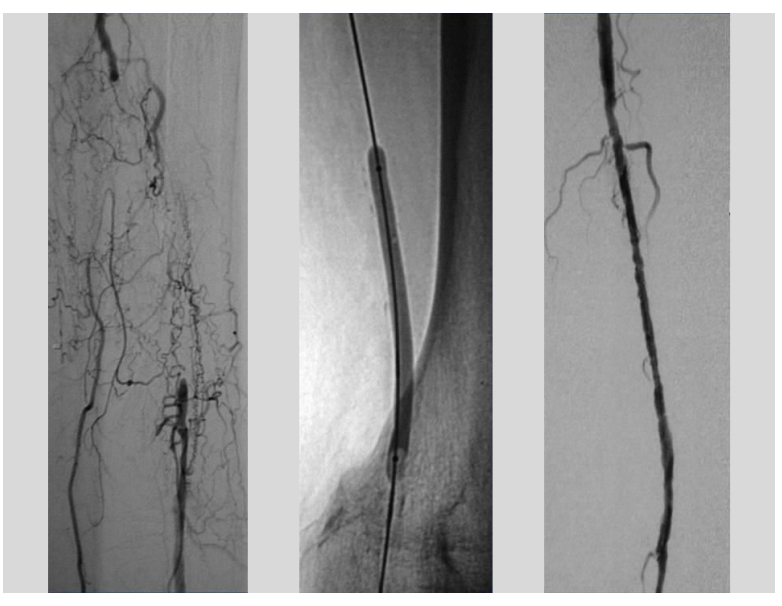

Figure 3: (A): Angiography demonstrated diffuse atherosclerotic disease with a long segment superficial femoral artery occlusion. (B): Angioplasty was performed using a $6 \mathrm{~mm}$ non-compliant angioplasty balloon. (C): Subsequent angiography shows resolution of the occlusion.

Patients without contraindications for anti-platelet therapy receive aspirin or clopidogrel following PTA. Aspirin is continued indefinitely unless contraindicated. Clopidogrel can also be given for 30 days post procedurally or indefinitely as part of a dual anti-platelet regimen in select patients. Our routine is aspirin for life and one month clopidogrel for bare metal stents. If newer drug eluting stents are used, six months to one year dual anti-platelet therapy is ideal. The risks and benefits must be weighed, and the risk of hemorrhage should be considered. Routine anti-coagulation is not needed. Cardiovascular risk factor modification, including smoking cessation and statin therapy must be continued. This treatment is important to maintain arterial patency after revascularization. More importantly, this therapy will reduce the patients overall cardiovascular morbidity and mortality.

\section{PTA \& CLI}

The clearest indication for performing revascularization is CLI. CLI results in significant morbidity and mortality regardless of the intervention performed [3]. In the BASIL trial, $80 \%$ of patients treated for CLI had SFA angioplasty. In this cohort, PTA was found to have a similar amputation free survival and overall survival with less morbidity and a reduced overall health care cost for up to 2 years [10].
After 2 years however, bypass surgery offers a 5.9 month amputation free survival advantage and a 7.3 month overall survival advantage [11]. The BASIL survival prediction model introduced by the BASIL investigators suggests that for patients with a 2 year predicted survival of $\geq 90 \%$, bypass surgery is preferable. On the other hand, if the 2 year predicted survival is $\leq 10 \%$, PTA is favorable [12]. For patients with a predicted survival between $10 \%$ and $90 \%$, the choice of treatment is based on the disease pattern, patient characteristics, and surgeon comfort and preference. Also resulting from the BASIL trial, Forbes et al demonstrated that the 3 year cost and health-related quality of life (HRQOL) were similar between surgical bypass and angioplasty [13].

\section{PTA vs Stenting}

Early randomized controlled trials assessing the efficacy of stenting using stainless steel stents failed to demonstrate an advantage over angioplasty alone $[3,14,15]$. However, there have been several newer studies comparing PTA to Nitinol stents in the SFA (Figure 4). These studies indicate that angioplasty alone results in equivalent patency rates when compared to primary stenting in patients with short stenoses or occlusions. Alternatively, longer stenoses or occlusions are best treated with primary stenting.

The FAST trial, a multi-center RCT, compared SFA PTA and Nitinol stenting in 244 patients [16]. The indication to treat was claudication in $97 \%$ of both groups. The mean lesion length treated in the study was shorter, $4.4 \mathrm{~cm}$ in the stenting group and $4.5 \mathrm{~cm}$ in the PTA group. Mortality and amputation rates were comparable. No significant differences in the re-stenosis rates (38.6\% in the PTA group vs. $31.7 \%$ in the stent group) or change in Rutherford criteria was observed between groups. Absolute walking distance (based on a treadmill test) was better in the PTA group (52 meters vs. 20 meters, $\mathrm{p}=0.028$ ) though the ankle-brachial indices (ABIs) remained similar at 12 months.

Schillinger et al. in a single center, randomized controlled trial (RCT), compared SFA PTA to Nitinol stenting in 104 patients [17]. This population was treated for intermediate lesion lengths, $13.2 \mathrm{~cm}$ in the stenting group and $12.7 \mathrm{~cm}$ in the PTA group. The indication for treatment was claudication in the majority of cases, $88 \%$ and $87 \%$ in the stenting and PTA groups, respectively. The groups did not differ in limb salvage or mortality. On an intention-to-treat and as-treated basis, the re-stenosis rate was greater for PTA ( $43 \%$ vs. $24 \%, \mathrm{p}=0.05$ and $50 \%$ vs. $25 \%, \mathrm{p}=0.02$, respectively). Duplex ultrasound at 12 month also demonstrated a greater re-stenosis rate $(63 \%$ vs. $37 \%$, $\mathrm{p}=0.01$ ). Moreover, the maximal walking distance was significantly less in the PTA group at 6 and 12 months (at 12 months: 267 meters vs. 387 meters, $\mathrm{p}=0.04$ ).

The RESILIENT trial is a second multi-center RCT that compared SFA PTA to Nitinol stenting in 206 patients [18]. The mean lesion length treated in this study was $7.7 \mathrm{~cm}$ in the stent group and $6.4 \mathrm{~cm}$ in the PTA group. The indication for treatment was claudication. Major adverse events did not differ between groups. In an intention-to-treat analysis, primary patency was worse in the PTA group at 6 months $(47.4 \%$ vs. $94.2 \%, \mathrm{p}<0.0001)$ and 12 months $(36.7 \%$ vs. $81.3 \%$, $\mathrm{p}<0.0001)$. In addition, angioplasty had inferior primary patency at 6 months $(79.3 \%$ vs. $94.4 \%, \mathrm{p}=0.03)$ and 12 months $(61.5 \%$ vs. $80.4 \%$, $\mathrm{p}=0.03)$ on the as-treated analysis.

Overall, when comparing PTA with provisional stenting to primary Nitinol stenting as an initial treatment, Nitinol stenting appears to have better patency at 6 and 12 months for intermediate to long length 
lesions with an improved walking distance (Figure 4). For short lesions, PTA appears to provide equivalent patency rates and may improve walking distance more than primary Nitinol stenting. Nevertheless, some authors have advocated PTA alone even for long lesions citing no difference between subintimal PTA and stenting using a stent graft [7].

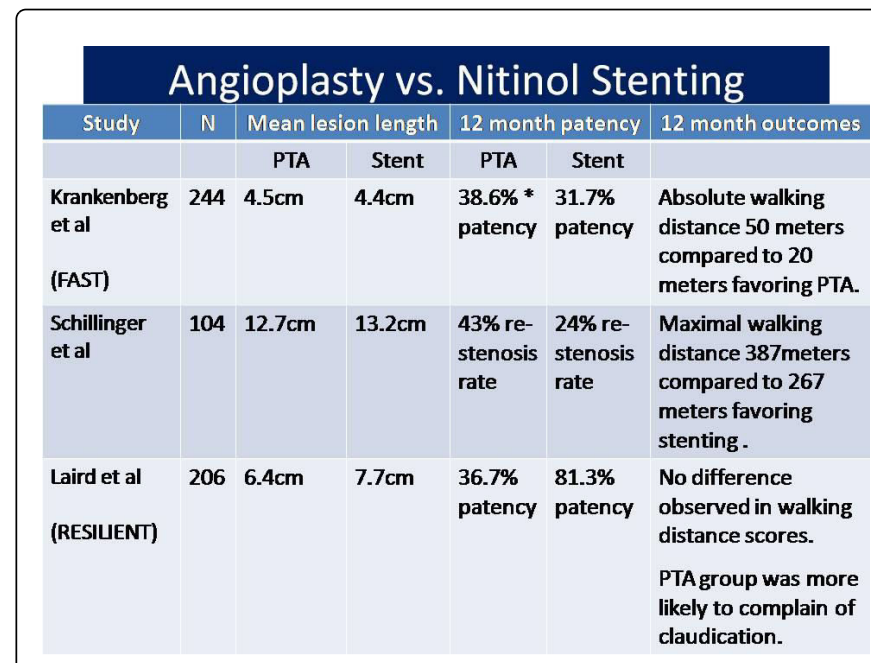

Figure 4: Summary of randomized clinical trials comparing angioplasty to stenting using Nitinol stents. PTA, Percutaneous Transluminal Angioplasty $\left({ }^{\star} \mathrm{p}=\mathrm{NS}\right)$

\section{Conclusion}

Endovascular therapy is an effective revascularization option in appropriately selected patients with SFA occlusive disease who present with debilitating claudication or CLI. Once indicated, this therapy begins by identifying the target stenosis or occlusion angiographically. The offending lesion must be traversed using a combination of a guide catheter and guide wire. After crossing the lesion, PTA is performed using an appropriately selected angioplasty balloon catheter. Subsequently, an angiogram is completed to assess the response to treatment. When a residual stenosis or flow limiting dissection is visualized, stenting is necessary. Current literature favors the use angioplasty for short SFA stenoses or occlusions. However, primary stenting using Nitinol stents may be a superior initial treatment for intermediate and long length lesions.

\section{References}

1. DOTTER CT, JUDKINS MP (1964) TRANSLUMINAL TREATMENT OF ARTERIOSCLEROTIC OBSTRUCTION. DESCRIPTION OF A NEW TECHNIC AND A PRELIMINARY REPORT OF ITS APPLICATION. Circulation 30: 654-670.

2. Grüntzig AR, Senning A, Siegenthaler WE (1979) Nonoperative dilatation of coronary-artery stenosis: percutaneous transluminal coronary angioplasty. N Engl J Med 301: 61-68.
3. Norgren L, Hiatt WR, Dormandy JA, Nehler MR, Harris KA, et al. (2007) Inter-Society Consensus for the Management of Peripheral Arterial Disease (TASC II). J Vasc Surg 45 Suppl S: S5-67.

4. Rutherford RB, Baker JD, Ernst C, Johnston KW, Porter JM, et al. (1997) Recommended standards for reports dealing with lower extremity ischemia: revised version. J Vasc Surg 26: 517-538.

5. Nowygrod R, Egorova N, Greco G, Anderson P, Gelijns A, et al. (2006) Trends, complications, and mortality in peripheral vascular surgery. $\mathrm{J}$ Vasc Surg 43: 205-216.

6. Pitta SR, Prasad A, Kumar G, Lennon R, Rihal CS, et al. (2011) Location of femoral artery access and correlation with vascular complications. Catheter Cardiovasc Interv 78: 294-299.

7. Boufi M, Dona B, Orsini B, Auquier P, Hartung O, et al. (2010) A comparison of the standard bolia technique versus subintimal recanalization plus Viabahn stent graft in the management of femoropopliteal occlusions. J Vasc Surg 52: 1211-1217.

8. Markose G, Miller FN, Bolia A (2010) Subintimal angioplasty for femoropopliteal occlusive disease. J Vasc Surg 52: 1410-1416.

9. London NJ, Srinivasan R, Naylor AR, Hartshorne T, Ratliff DA, et al. (1994) Subintimal angioplasty of femoropopliteal artery occlusions: the long-term results. Eur J Vasc Surg 8: 148-155.

10. Adam DJ, Beard JD, Cleveland T, Bell J, Bradbury AW, et al. (2005) Bypass versus angioplasty in severe ischaemia of the leg (BASIL): multicentre, randomised controlled trial. Lancet 366: 1925-1934.

11. Bradbury AW, Adam DJ, Bell J, Forbes JF, Fowkes FG, et al. (2010) Bypass versus Angioplasty in Severe Ischaemia of the Leg (BASIL) trial: An intention-to-treat analysis of amputation-free and overall survival in patients randomized to a bypass surgery-first or a balloon angioplastyfirst revascularization strategy. J Vasc Surg 51: 5S-17S.

12. Bradbury AW, Adam DJ, Bell J, Forbes JF, Fowkes FG, et al. (2010) Bypass versus Angioplasty in Severe Ischaemia of the Leg (BASIL) trial: A survival prediction model to facilitate clinical decision making. J Vasc Surg 51: 52S-68S.

13. Forbes JF, Adam DJ, Bell J, Fowkes FG, Gillespie I, et al. (2010) Bypass versus Angioplasty in Severe Ischaemia of the Leg (BASIL) trial: Healthrelated quality of life outcomes, resource utilization, and costeffectiveness analysis. J Vasc Surg 51: 43S-51S.

14. Grimm J, Müller-Hülsbeck S, Jahnke T, Hilbert C, Brossmann J, et al. (2001) Randomized study to compare PTA alone versus PTA with Palmaz stent placement for femoropopliteal lesions. J Vasc Interv Radiol 12: 935-942.

15. Muradin GS, Bosch JL, Stijnen T, Hunink MG (2001) Balloon dilation and stent implantation for treatment of femoropopliteal arterial disease: meta-analysis. Radiology 221: 137-145.

16. Krankenberg H, Schlüter M, Steinkamp HJ, Bürgelin K, Scheinert D, et al. (2007) Nitinol stent implantation versus percutaneous transluminal angioplasty in superficial femoral artery lesions up to $10 \mathrm{~cm}$ in length: the femoral artery stenting trial (FAST). Circulation 116: 285-292.

17. Schillinger M, Sabeti S, Loewe C, Dick P, Amighi J, et al. (2006) Balloon angioplasty versus implantation of nitinol stents in the superficial femoral artery. N Engl J Med 354: 1879-1888.

18. Laird JR, Katzen BT, Scheinert D, Lammer J, Carpenter J, et al. (2010) Nitinol stent implantation versus balloon angioplasty for lesions in the superficial femoral artery and proximal popliteal artery: twelve-month results from the RESILIENT randomized trial. Circ Cardiovasc Interv 2010. 